\title{
Cognitive Radio Systems: Multicarrier Modulation and Power Allocation Challenges
}

Ahmed Sohail ${ }^{*}$, Mohammed Al-Imari, Pei Xiao and Barry G. Evans

Centre for Communication Systems Research, University of Surrey, Guildford, GU2 7XH, United Kingdom

\begin{abstract}
The rapid development of modern communication services results in high data rate requirements from the end user. It is challenging to meet high data rate requirements because of prevailing issues such as spectrum scarcity and spectrum underutilization due to fixed spectrum assignment policy. Cognitive Radio (CR), being the enabler of dynamic spectrum management techniques, has the capability to tackle these issues by proficiently implementing spectrum sharing schemes using Multicarrier Modulation (MCM) techniques. In CR system, where the Primary User (PU) and the Secondary User (SU) co-exist in the same frequency band, mutual interference (i.e., from SU to PU and vice versa) is a limiting factor on the achievable capacity of both the PU and the SU. Power allocation in MCM based CR systems aims to dynamically control the transmit power on each subcarrier of the $\mathrm{SU}$ in order to reduce the mutual interference. Furthermore, combining multiple antennas with MCM is regarded as a very attractive solution for the CR communications to effectively enhance data rate without demanding additional bandwidth and transmit power.
\end{abstract}

Keywords: Cognitive radio; Multicarrier modulation techniques; Power allocation

\section{Introduction}

Radio spectrum is a scarce resource and its demand is growing rapidly to meet high data rate end user demands. Current static frequency allocation leads to insufficient usage of limited spectrum resources and urges the regulatory bodies to review their policy and inquire communication technologies that can exploit spectrum in a more flexible and intelligent fashion. For example, spectrum occupancy measurements conducted by Ofcom in different areas of UK, show underutilization of spectrum in significant periods of time. Similarly, according to FCC, in New York City and downtown Washington DC only $13.1 \%$ and $35 \%$ of spectrum utilization has been reported, respectively, below $3 \mathrm{GHz}$ [1]. These studies clearly suggest that physical spectrum shortage is mainly due to the inflexible spectrum licensing scheme.

Cognitive Radio (CR) is an emerging technology that has the potential to significantly improve the spectrum efficiency. It allows the Secondary User (SU) to opportunistically access the licensed spectrum given an acceptable interference to the Primary User (PU) [2]. The key idea behind CR technology is to enhance the spectrum efficiency and maximize the data rate through four possible spectrum sharing schemes; (i) Underlay Spectrum Sharing (USS) scheme, (ii) Overlay Spectrum Sharing (OSS) scheme, (iii) Interweave (opportunistic) Spectrum Sharing (ISS) scheme, (iv) Mixed Spectrum Sharing (MSS) scheme. The performance of the aforementioned schemes depends on the CR environment.

To address associated challenges of CR system, Multicarrier Modulation (MCM) techniques are the potential candidates. As of today, Orthogonal Frequency Division Multiplexing (OFDM) system has been proposed as a mature and dominant technology in CR systems due to its flexibility, simple equalization enabled by the use of a Cyclic Prefix (CP) and resistant to narrowband interference. However, the reduced power and spectral efficiency due to the use of $\mathrm{CP}$, generation of large side-lobes and its sensitivity to frequency offset, raise questions on OFDM candidacy in CR systems. Furthermore, aforementioned drawbacks also have a direct impact on the achievable capacity of the SU. On the other hand, Filter Bank Multicarrier (FBMC) system achieves higher spectral efficiency due to the fact that $\mathrm{CP}$ is no longer needed. Furthermore, it offers full control over spectral leakage as a result of its improved spectral shape at a cost of higher implementation complexity and latency compared to OFDM systems [3].

In CR system, another associated challenge is the mutual interference which is a capacity limiting factor for both the PU and the SU networks. The amount of interference introduced by the SU subcarriers into the PUs band depends on three factors, i.e., power allocated in that subcarrier, spectral distance between that particular subcarrier and the PUs band, and location of the PU (whether it is detectable or not by the SU). To address this issue, different power allocation schemes have been proposed in the literature where Gaussian inputs are assumed to maximize the $\mathrm{SU}$ data rate for a given interference threshold values $[4,5]$. However, the Gaussian input assumption is unrealistic, whereas, Finite Symbol Alphabet (FSA) input is more applicable to practical systems. To determine the difference between the Gaussian and the FSA input, a Signal-to-Noise Ratio (SNR) gap model has been proposed in [6], where the achievable rates attained by the FSA input are approximated by the capacity attained by the Gaussian input. However, this approach is not valid at high SNRs due to the large gap and its inability to predict the rate saturation point. In Lozano, et al. [7] and Zeng, et al. [8], authors derived optimal power allocation using the FSA input in a non-cognitive scenario, whereas in an interference limited CR system, the same power allocation algorithms cannot be applied due to mutual interference. Therefore, in Sohail, et al. $[9,10]$, an optimal power scheme given an FSA input distribution is derived for a case of single and multiple antenna techniques. A significant transmit power can be saved when the optimal power derived under the FSA

*Corresponding author: Ahmed Sohail, Centre for Communication Systems Research, University of Surrey, Guildford, GU2 7XH, United Kingdom, Tel: 0148368 3600; E-mail: a.sohail@surrey.ac.uk

Received July 3, 2013; Accepted October 25, 2013; Published October 28, 2013

Citation: Sohail A, Al-Imari M, Xiao P, Barry G, Evans (2013) Cognitive Radio Systems: Multicarrier Modulation and Power Allocation Challenges. J Electr Electron Syst 3: 116. doi:10.4172/2332-0796.1000116

Copyright: ( 2013 Sohail A, et al. This is an open-access article distributed under the terms of the Creative Commons Attribution License, which permits unrestricted use, distribution, and reproduction in any medium, provided the original author and source are credited. 
input is used for FSA transmission compared to the optimal power under the Gaussian input. Moreover, Gaussian optimized power results in a reduced transmission rate due to extra allocated power causing nulling of more subcarriers compared to the optimal power under the FSA input.

\section{Spectrum sharing schemes in CR system}

Spectrum sharing alleviates the spectrum looming problem. It enhances the spectrum efficiency by allowing SU to access a frequency band formally allocated to the PU under certain conditions without imposing any changes to the primary network or inter-system signalling. For efficient spectrum sharing, SU should be aware of the PU characteristics, e.g., air interface, transmit power, bandwidth, knowledge about spectrum and channel conditions. Following are different spectrum sharing schemes in CR system.

\section{Underlay Spectrum Sharing (USS) scheme}

The USS scheme does not require sensing and SU can always access the PU spectrum simultaneously in the spectrum band of interest under the condition that the interference introduced by the SU is below the acceptable noise floor of the PUs [11]. In this scheme, the data rate is independent of PU activity however, the SU transmit power is low. It guarantees low data rate and is suitable for short range applications.

\section{Interweave Spectrum Sharing (ISS) scheme}

On the contrary to USS scheme, sensing is required in the ISS scheme and the spectrum is only accessible by the SU once the PU is idle [11]. Therefore, the data rate is dependent on the spectrum sensing results and the PU activity. Imperfect spectrum sensing leads to missed detection and false alarm. In missed detection the channel is wrongly considered idle, causing collisions between the PU and the SU while false alarm makes the SU keep silence even if the idle channel is available to SU [12]. The ISS scheme can provide high data rate (when PU activity is less) but without any guarantee compared to the USS scheme and is suitable for wide range applications.

\section{Overlay Spectrum Sharing (OSS) scheme}

The OSS scheme also allows simultaneous transmission of the PU and the SU; however, the SU can use part of its power for secondary transmission and the remaining power for primary transmission in order to compensate the PU's Signal-to-Noise Ratio (SNR) degradation. The main limitation of the OSS scheme is that it requires a priori knowledge of the PU's transmission. Furthermore, it works well when primary and secondary transmitters are in close proximity [11].

\section{Mixed Spectrum Sharing (MSS) scheme}

The main limitation of USS and ISS schemes is that in the USS scheme, capacity can be further increased when the PU is not active (idle), however it is still required by the SU to satisfy the Interference Temperature Constraint (ITC). During idle periods, ITC puts an upper bound on transmission power of the SU, thus limiting its achievable capacity. On the other hand, in the ISS scheme SU cannot access the spectrum during busy periods and ignores the opportunity of spectrum accessibility by imposing ITC. Therefore, either in ISS or USS scheme the spectrum cannot be fully exploited. To address this problem MSS scheme is proposed in order to utilize the spectrum more efficiently [4]. Unlike the USS scheme, SU in the MSS scheme transmits without taking the ITC into consideration during idle periods and ITC is imposed during busy periods in contrast to the ISS scheme. The MSS scheme achieves and guarantees higher capacity compared to ISS and
USS since it fully exploits the limited available spectrum, but at a cost of higher complexity.

\section{Switch access scheme}

The channel capacity of the $\mathrm{SU}$ is directly proportional to the $\gamma$, i.e., probability of the SU access to the primary spectrum. In the USS scheme, primary spectrum is always accessible to the SU, therefore channel capacity is independent of the $\gamma$ and the same achievable rate is guaranteed regardless of the PU traffic. However, in the ISS scheme where the spectrum is only accessible by the SU once it is left by the PU, the achievable rate cannot be guaranteed due to the dependency on $\gamma$. Based on $\gamma$, a switch access scheme (between ISS and USS) is proposed in [4]. When $\gamma$ is small, USS schemes achieves higher capacity than the ISS scheme (even zero at $\gamma=0$ ), while ISS scheme outperforms USS scheme when $\gamma$ is high.

\section{Challenges in CR System}

This section will highlight some challenges in CR system especially by applying MCM techniques and power allocation algorithms in different spectrum sharing schemes.

\section{Challenges due to MCM techniques in CR system}

In current wireless communication standards and services, MCM techniques are widely used due to its mitigation of multipath [13]. It is also very suitable for CR systems because it has the capability to monitor the PU spectral activity and flexibility to dynamically allocate unused licensed spectrum among SU subcarriers [14]. However, there exist some challenges by applying MCM techniques in CR system which needs to be properly addressed for interference free co-existence between PU and SU.

In OFDM system, transmit prototype filter yields in large side-lobes, e.g., the difference between the peaks of the first side-lobe and the main lobe is only $13 \mathrm{~dB}[3]$. These side-lobes cause signal power leakage into neighboring subcarriers, leading to a high sensitivity to frequency offsets causing interference to the neighboring users. The problem is more severe in CR systems where the SU is not allowed to cause interference to the PU and both have different access technologies. To overcome this problem, either both schemes must be perfectly synchronized or side-lobe suppression techniques are required. Perfect synchronization in CR system is difficult to achieve due to lack of cooperation between the PU and the SU and side-lobe suppression techniques are at the cost of complexity. On the other hand, in FBMC systems, side-lobes are avoided due to the use of near perfect filters. Therefore, higher channel capacity can be achieved in FBMC systems as they offer full control over spectral leakage compared to OFDM systems.

Furthermore, in uplink scenario perfect synchronization is not possible in practical OFDM systems due to the mobility of terminals. Interference cancellation techniques are required to minimize mutual access interference, which significantly increases the receiver's complexity. On contrary, the situation is less severe in FBMC system as it uses near perfect filters, thus, avoiding the need for any interference cancellation techniques $[3,15]$.

\section{Challenges due to power allocation algorithms in different spectrum sharing schemes}

Transmission power is an important resource in wireless communication systems, especially in co-existence scenarios where the time-varying nature of the channels and interference are limiting factors for network performance. Efficient power allocation algorithms 
serve many purposes, (e.g., reducing interference, maintaining data quality, maximizing cell capacity, etc.). Power allocation in MCM based CR system aims to control the transmit power on each subcarrier of the SU dynamically in order to achieve maximum SU capacity without causing interference to the PU.

Power allocation in ISS scheme: In conventional OFDM systems, power allocation mainly depends on the channel gain of the subcarriers. If the channel condition is good, more power is allocated to that subcarrier and vice versa. However, the same power allocation scheme cannot be applied to MCM based CR systems due to mutual interference. The level of interference introduced to the PU's band not only depends on the power allocated in that subcarrier, but also depends on the spectral distance between that particular subcarrier and the PUs band. Therefore, in the interference limited scenario, allocation of power is based on the position of the subcarrier with respect to the PU's spectrum, i.e., more power should be allocated to distant subcarriers and vice versa. Therefore, in MCM based-CR systems, a judicious power loading scheme is required which should take into consideration the channel gain of the subcarrier as well as the spectral distance between the subcarrier and the PUs band [5]. When the same power allocation scheme is employed in both FBMC and OFDM systems, FBMC achieves higher capacity due to absence of $\mathrm{CP}$ which allows more available subcarriers and full control over spectral leakage [16].

Another important aspect of power allocation in CR systems is the reliability of the subcarriers, (i.e., subcarriers that are more frequently available for SU transmission as compared to those which are always busy due to the PU activity). Previously, it was assumed that after sensing, spectrum holes are available to the secondary transmission up to a certain time until the SU completes its task. However, in the real time scenario, the PU being the spectrum owner may return at any time and retrieve its spectrum which is currently available for the secondary access. Therefore, power allocated by the SU is wasted due to the unaccomplished task by the SU. In view of this fact, more power should be allocated to more reliable subcarriers in order to guarantee the SU's QoS requirements [17,18].

Power allocation in USS scheme: Unlike ISS, the USS scheme imposes severe transmission power constraints on the SUs due to concurrent access of the spectrum by both the PU and the SU. Therefore, in order to maximize the sum capacity in the CR system, a modified water-filling algorithm, (e.g., iterative partitioned water filling algorithm) with the consideration of per sub-channel power constraint in addition to sum power constraint is proposed in Wang, et al. [19].

Power allocation in MSS scheme: By allocating transmission power to either the ISS or the USS scheme, the entire degree of freedom cannot be achieved. In the ISS scheme, the total transmit power is allocated to the interweave subcarriers. However, it might be possible that the underlay subcarriers experience very good channel conditions compared to the interweave subcarriers and can achieve higher channel capacity. Therefore, an optimal scheme for capacity maximization has been introduced which allocates power to both the underlay and the interweave subcarriers. In this mixed scheme, more power is allocated to the interweave subcarriers and less power to the underlay subcarriers since underlay subcarriers introduce more interference to the PU band. This ensures that the interference introduced to the PU bands is below a prescribed threshold but also maintains a total transmission power within the budget [20].

\section{Practical Consideration for Power Allocation}

In literature, optimal power assuming the Gaussian input distribution has been investigated in CR systems to maximize the capacity of the SU while keeping the interference introduced to the PU band within the tolerable range [5,17]. However, the Gaussian input assumption is unrealistic, whereas practical systems use FSA input distributions, (i.e., M-QAM). To this end, optimal power under the condition of FSA input is considered to achieve practical data rates. With this power loading scheme, achievable data rates saturate at high SNR contrary to the optimal power under the Gaussian input. The reason for this saturation is that the achievable rate attained by the FSA input is bounded in the SNR. Thus beyond the saturation point, the achievable rate remains constant regardless of the SNR values. In Sohail, et al. [21], the effect of SNR value on the saturation point is investigated in both cognitive and non-cognitive scenarios. It has been found that the CR system achieves saturation point after the noncognitive scenario, however, the maximum achievable data rate after the saturation point remains constant for both scenarios with the FSA input distribution.

\section{For SISO case}

Optimal power and achievable data rate under Gaussian and FSA inputs are compared in Sohail, et al. [9] for a single-input single-output (SISO)-OFDM based CR network via the ISS scheme.

System model: The system model consists of a single-cell wireless system in the downlink path, where the PU and the SU transceivers co-exist in the same geographical location as shown in Figure 1. The instantaneous fading gains considered in the simulations are listed as follows, and are assumed to be known a-priori at the SU transmitter via a pilot-assisted channel estimation algorithm: (i) $g_{n}^{s S}$, between the SU transmitter and SU receiver for the $n_{t h}$ subcarrier; and (ii) $g_{m}^{s p}$, between the SU transmitter and $m_{t h}$ PU receiver.

As shown in Figure 2, the co-existence of a PU and a SU in the frequency domain is considered, where the user data are mapped to consecutive subcarriers. In the ISS scheme, due to adjacent co-existence of the PU and the SU, two types of interference have been introduced in the system. One is introduced from the PU into the SU band, and the other is introduced from the SU into the PU band. The objective is to protect the PU from unacceptable interference, therefore, only interference introduced by the SU into the PU band is considered [5] and interference introduced by the PU into the SU is treated as a noise

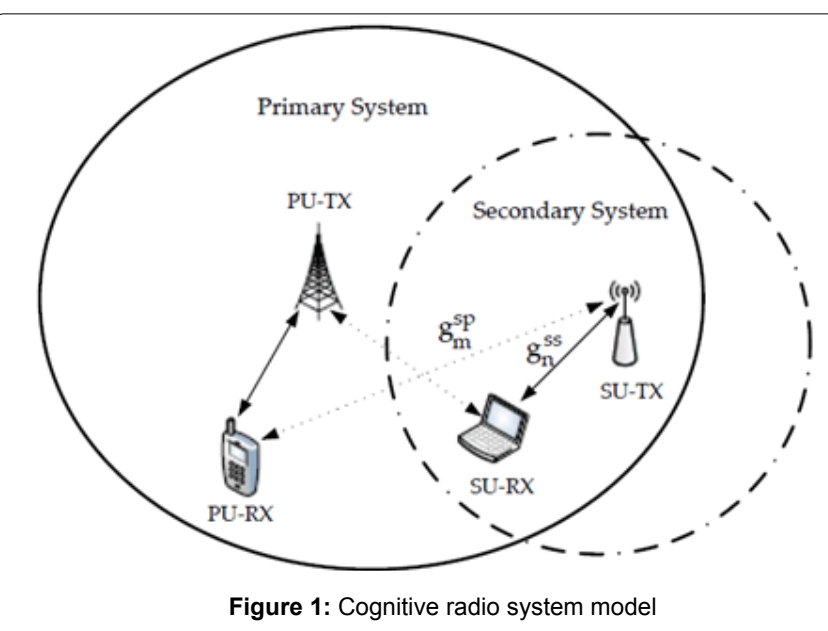


[9].

Simulation parameters: For practical reasons, LTE parameters are adopted and assumed that the available bandwidth for the SU transmission is $10 \mathrm{MHz}$ which is divided into 50 resource blocks (RBs) [22]. A simplified path loss model, i.e., $Q\left(r_{d} / r\right)$ [23] is considered for simulations, where $Q$ is constant, $r_{0}$ is reference distance and $r$ is the distance between the SU transmitter and the PU receiver in meters. The values of symbol period, (i.e., $T_{s}$ ) and $r_{0}$ are $4 \mathrm{~ms}$ and 50 meters, and $\tau_{\text {th }}$ is assumed to be equivalent to thermal noise per $\mathrm{RB}$, respectively. The value of $\tau_{\text {th }}$ increases according to $r$ and in the simulation, $r$ ranges from 50 to 85 meters. The IEEE 802.11 multipath channel model with root mean square delay spread of $50 \mathrm{~ns}$ is assumed. The results are averaged over 2000 snapshots. Total transmit optimal power with the Gaussian input is represented as $P_{G}^{*}$ and with the FSA input, (i.e., BPSK, $Q P S K$, 16-QAM) as $P_{F}^{*}$.

Simulation results: Figure 3 shows the comparison of $P_{G}^{*}$ and $P_{F}^{*}$ versus distance. It can be observed that $P_{G}^{*} \mathrm{i}$ always greater than $P_{F}^{*}$ over the considered distance range. It has further been noticed that the power difference gap increases in proportion to the distance metric values, meaning that the power difference gap is smaller at lower distance values compared to higher distance values. This could be explained as: (i) the increase in $P_{F}^{*}$ is marginal at higher distance values because the Mutual Information (MI) reaches an upper bound limit; (ii) on the other hand, $P_{G}^{*}$ increases with increasing distance values because the MI under $P_{G}^{*}$ has no upper bound limit. Moreover, it has been observed that at a fix distance metric value, $P_{F}^{*}$ increases with increasing modulation scheme, (i.e., from BPSK to 16-QAM). The optimal power allocation is dependent and specific for every modulation scheme. It would result in power inefficiency if one tries to transmit BPSK signal with the power which is optimized for 16-QAM. Therefore, for efficient power

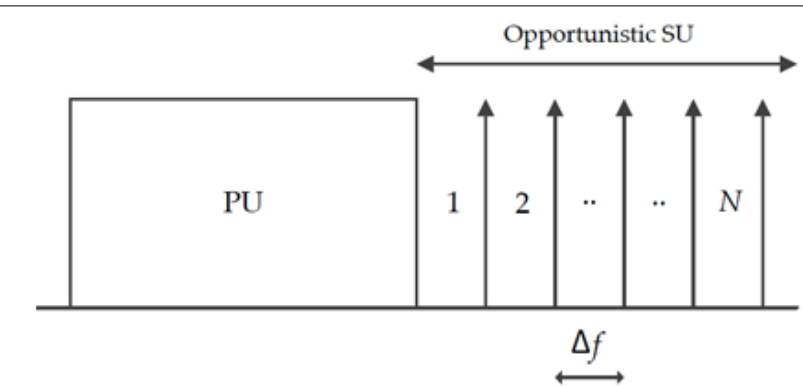

Figure 2: Graphical representation of interweave spectrum sharing scheme

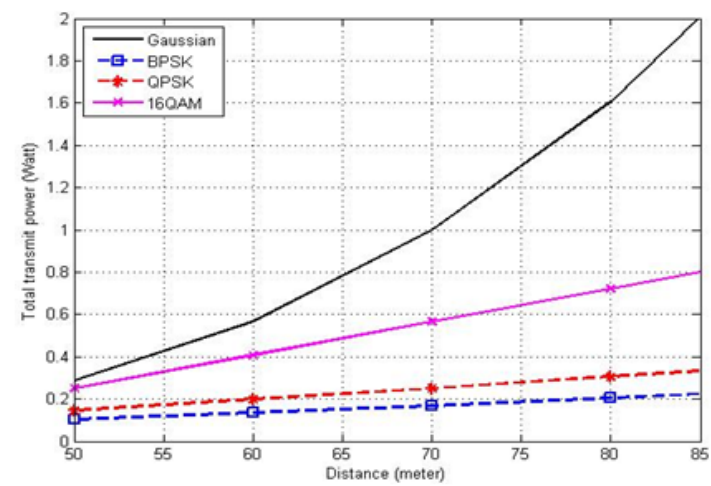

Figure 3: Optimal Power under Gaussian and FSA inputs vs. distance utilization, power must be optimized according to the actual employed modulation scheme.

Power saving (i.e., $\left.\frac{P_{G}^{*}-P_{F}^{*} * 100 \%}{P_{G}^{*}}\right)$ for BPSK, QPSK and 16-QAM versus distance is demonstrated in Figure 4. It can be clearly seen that a significant power saving has been achieved by the $P_{F}^{*}$ in comparison to the $P_{G}^{*}$. The transmit power saving for distance values ranging from 50 $\mathrm{m}$ to $85 \mathrm{~m}$ has found out to be $65-90 \%, 50-82 \%$ and $12-60 \%$ for BPSK, QPSK and 16-QAM inputs, respectively.

Figure 5 shows a comparison of achieved data rate for the FSA transmission between the Gaussian optimized power and the power optimized based on the actual modulation scheme. It can be seen that the proposed optimal power allocation scheme achieves higher data rates compared to the traditional Gaussian power allocation scheme.

In Figure 6, the impact of distance metric ranging from $50 \mathrm{~m}$ to 85 $\mathrm{m}$ on percentage of rate gain for the CR system has been shown. The BPSK, QPSK and 16QAM inputs achieve a rate gain of 31-24\%, 24-19\% and $6-10.5 \%$, respectively. The higher interference caused by the coexistence of PU and SU in the CR system limits the percentage rate gain for the Gaussian optimized power. It is because as $P_{G}^{*}$ becomes higher than the $P_{F}^{*}$, it consequently nullify more subcarriers in comparison to $P_{F}^{*}$ and thus more subcarriers are wasted. This allows the FSA input to achieve a better performance in terms of overall rate gain.

\section{For MIMO case}

Multiple antenna techniques are special case of conventional single antenna technique where transmitter and receiver are equipped with multiple antennas at both ends. It can significantly improve the spectrum efficiency and capacity of a system without requiring additional bandwidth and transmission power. The MIMO channel can be decomposed into parallel independent sub-channels by singular value decomposition. It decomposes the channel matrix into the product of three matrices; an orthogonal matrix $U$, a diagonal matrix $S$, and the transpose of an orthogonal matrix $V$, i.e.,

\section{$H=U S V^{*}$,}

where $U$ and $V$ are unitary matrices and $S$ is a diagonal matrix containing non-negative ordered eigen values of $H^{*}$. The columns of $U$ are eigenvectors vectors of $\mathrm{HH}^{*}$ and the columns of $\mathrm{V}$ are eigenvectors of $H^{\star} H$. In Sohail, et al. [10], the results of SISO case are extended for MIMO case with the same assumption made in Sohail, et al. [9]. It is observed from results of Sohail, et al. [10] that the achievable data rate of $2 \times 2$ CR systems is twice compared to single antenna CR system in Figure 2. It can be concluded that the achievable data rate is directly proportional to the number of transmit and receive antennas. However, the percentage of power saving and rate gain for single and multiple antennas case are the same. This is due to the fact that the optimal power and rate gain for Gaussian and FSA inputs increases with the same rate as the number of antenna increases.

\section{Conclusion and Future Work}

CR offers a solution to the spectrum underutilization problem by proficiently implementing spectrum sharing schemes using MCM techniques. The review draws several conclusions.

- Although, OFDM system is the most popular transmission scheme and the availability of low cost chip sets makes it potential candidate for CR systems, however, FBMC outperforms OFDM in terms of channel capacity due to better signaling shape.

- The performance achieved by different spectrum sharing schemes depends on the CR environment. Generally, it needs to be exploited that which spectrum sharing scheme is better than the other in terms of channel capacity. 


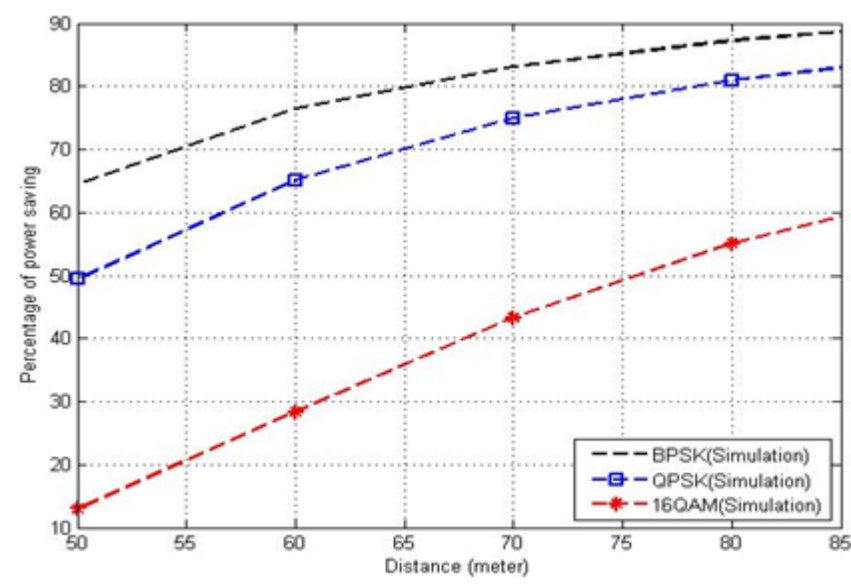

Figure 4: Percentage of power saving vs. distance

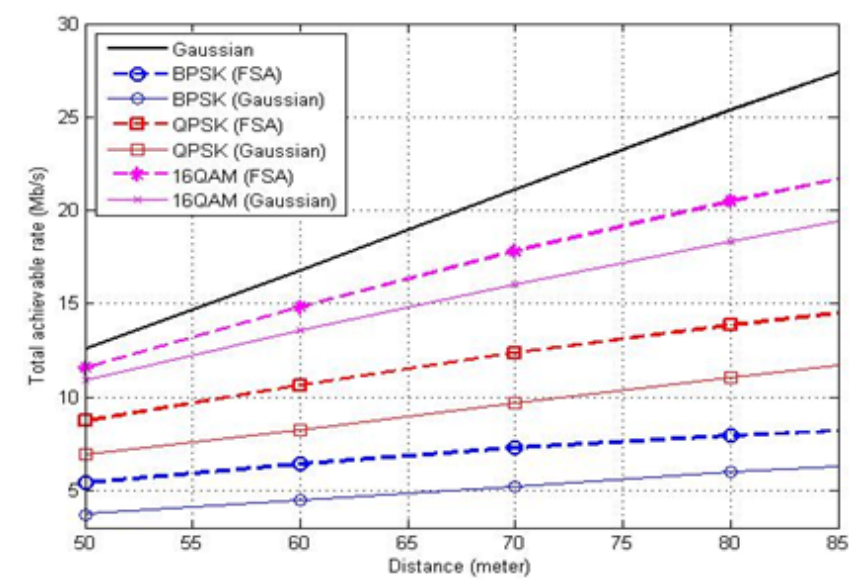

Figure 5: Achievable data rate under Gaussian and FSA inputs vs. distance

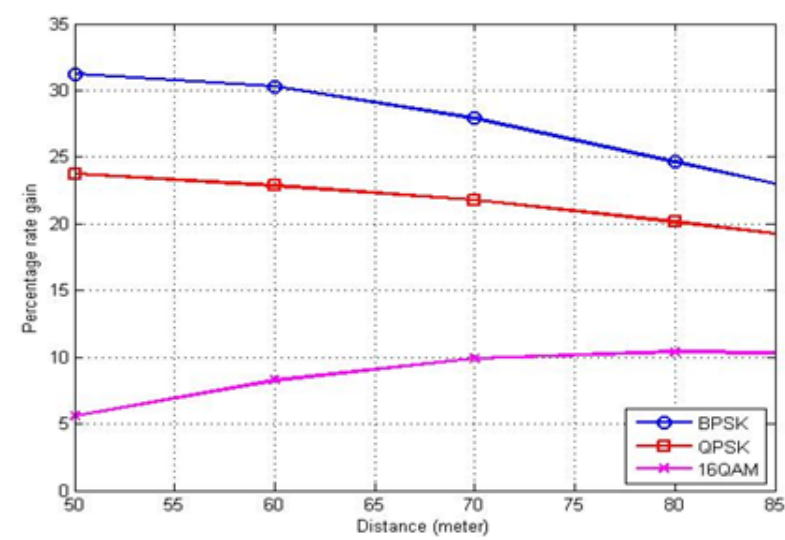

Figure 6: Percentage of rate gain vs. distance

- Optimal power allocation algorithms under Gaussian and FSA inputs are addressed in order to maximize the channel capacity. It has been shown that the optimal power with the FSA input significantly outperforms the optimal power with the Gaussian input in terms of transmit power saving and achievable data rate and consequently, spectrum and energy efficiency can both be improved.

- With fix distance metric, the optimal transmit power with the FSA input increases as the modulation order increases. Therefore, to achieve desired energy efficiency, the power should be optimized according to the employed modulation scheme.

- Although vast amount of research in spectrum underutilization has been done however, there are still many open problems remaining that need to be tackled. The possible future works for capacity maximization in CR systems are as follows:

- How to properly design FBMC based CR systems in conjunction with multiple antenna techniques for different spectrum sharing schemes in order to increase capacity deserves further investigation.

- In literature, optimal power allocation techniques in the CR system assume a static spectrum scenario, i.e., transmission period of SUs remain constant. However, in practical cases, PUs can arrive and depart from unoccupied and occupied bands, respectively, at any time irrespective of the transmission period of SUs, consequently causing interference to the PU. Therefore, dynamic spectrum scenario model should be investigated in order to achieve practical capacity.

- In Sohail, et al. $[9,10]$, optimal power under the FSA input has been evaluated for single cell assuming single SU. The optimal power under the FSA input can be investigated for multiuser scenario and multi cell environment.

- Since Authorized Shared Access (ASA) [24] offers pragmatic and fast track solution for regulators, ASA can be investigated in order to increase the efficient use of spectrum and to meet the ever increasing demand of spectrum.

Open access journals provide the platform for all researchers to make the freely available state of the art developments. OMICS group of publications is one of the platforms to share new research findings and make significant impact of ideas and solutions.

\section{References}

1. Arshad K, Imran MA, Moessner K (2010) Collaborative spectrum sensing optimisation algorithms for cognitive radio networks. International Journal of Digital Multimedia Broadcasting

2. Sendonaris A, Erkip E, Aazhang B (2003) User cooperation diversity. Part I. System description. IEEE T Commun 51: 1927-1938.

3. Farhang-Boroujeny B (2011) OFDM Versus Filter Bank Multicarrier. IEEE Signal Proc Mag 28: 92-112.

4. Khoshkholg MG, Navaie K, Yanikomeroglu H (2010) Access strategies for spectrum sharing in fading environment: Overlay, underlay, and mixed. IEEE Trans Mobile Computing 9: 1780-1793.

5. Bansal G, Hossain J, Bhargava VK (2008) Optimal and suboptimal power allocation schemes for OFDM-based cognitive radio systems. IEEE Transactions Wireless Communications 7: 4710-4718.

6. Devillers B, Louveaux J, Vandendorpe L (2008) Bit and power allocation for goodput optimization in coded parallel subchannels with ARQ. IEEE T Signal Proces 56: 3652-3661.

7. Lozano A, Tulino AM, Verdu S (2006) Optimum power allocation for paralle gaussian channels with arbitrary input distributions. IEEE T Inform Theory 52 3033-3051.

8. Zeng W, Chengshan X, Wang M, Lu J (2012) Linear precoding for finite alphabet inputs over MIMO fading channels with statistical CSI. IEEE T Signal Proces 60: 3134-3148.

9. Sohail A, Al-Imari M, Xiao P, Evans B (2013) Optimal power allocation fo OFDM based cognitive radio systems with arbitrary input distributions. IEEE Vehicular Technology Conference, 2013, Dresden, Germany. 
Citation: Sohail A, Al-Imari M, Xiao P, Barry G, Evans (2013) Cognitive Radio Systems: Multicarrier Modulation and Power Allocation Challenges. J Electr Electron Syst 3: 116. doi:10.4172/2332-0796.1000116

10. Sohail A, Al-Imari M, Xiao P, Evans B (2013) Optimal power allocation for MIMO-OFDM based cognitive radio systems with arbitrary input distributions. IEEE Personal, Indoor and Mobile Radio Communication Conference, 2013.

11. Srinivasa S, Jafar SA (2007) Cognitive radios for dynamic spectrum access - the throughput potential of cognitive radio: A theoretical perspective. IEEE Commun Mag 45: 73-79.

12. Tang W, Shakir MZ, Imran MA, Tafazolli R, Alouini M-S (2012) Throughput analysis for cognitive radio networks with multiple primary users and imperfect spectrum sensing. IET Communications 6: 2787-2795.

13. Mahmoud $\mathrm{H}$, Yucek T, Arslan $\mathrm{H}$ (2009) OFDM for cognitive radio: merits and challenges. IEEE Wireless Communications 16: 6-15

14. Weiss T, Hillenbrand J, Krohn A, Jondral FK (2004) Mutual interference in OFDM-based spectrum pooling systems. 59th IEEE Vehicular Technology Conference 4 : 1873-1877.

15. Du J, Xiao P, Wu J, Chen Q (2012) Design of isotropic orthogonal transform algorithm-based multicarrier systems with blind channel estimation. IET Communications 6: 2695-2704.

16. Shaat M, Bader F (2009) Low complexity power loading scheme in cognitive radio networks: FBMC capability. 20th IEEE Personal, Indoor and Mobile Radio Communications Symposium.
17. Hasan Z, Bansal G, Hossain E, Bhargava VK (2009) Energy-efficient power allocation in OFDM-based cognitive radio systems: A risk-return model. IEEE Transactions on Wireless Communications 8: 6078-6088.

18. Chen $\mathrm{CH}$, Wang $\mathrm{CL}$ (2010) Power allocation for OFDM-based cognitive radio systems under primary user activity. IEEE 71st Vehicular Technology Conference.

19. Wang P, Zhao M, and Xiao L, Zhou S, Wang J (2007) Power allocation in OFDM-based cognitive radio systems. IEEE Global Telecommunications Conference (GLOBECOM)

20. Bansal G, Duval O, Gagnon F (2010) Joint overlay and underlay power allocation scheme for OFDM-based cognitive radio systems. IEEE 71st Vehicular Technology Conference (VTC).

21. Sohail A, Xiao P Evans B (2012) Optimal power allocation scheme under FSA constraint for OFDM based cognitive radio systems. IEEE International Conference on Ultra Modern Telecommunications (ICUMT)

22. Zyren J, McCoy W (2007) Overview of the 3GPP long term evolution physical layer. Freescale Semiconductor Inc., white paper.

23. Goldsmith (2005) Wireless communications. Cambridge University Press, New York, USA.

24. Nokia Siemens Networks Policy and Government Relations on "Authorized Shared Access". 